\title{
Human Papilloma Virus-positive Oropharyngeal Squamous Cell Carcinoma in the Elderly
}

\author{
EESHA DAVE $^{1}$, WILLIAM SU ${ }^{1}$, VISHAL GUPTA ${ }^{2}$, BRETT MILES ${ }^{3}$, \\ ELIZABETH DEMICCO ${ }^{4}$, THERESA SORIANO ${ }^{5}$ and RICHARD L. BAKST ${ }^{2}$ \\ Departments of ${ }^{1}$ Medical Education, ${ }^{2}$ Radiation Oncology, ${ }^{3}$ Otolaryngology-Head and Neck Surgery, \\ ${ }^{4}$ Pathology, and ${ }^{5}$ Geriatrics and Palliative Medicine, \\ Icahn School of Medicine at Mount Sinai, New York, NY, U.S.A.
}

\begin{abstract}
Background/Aim: Elderly patients with HPV+ oropharyngeal cancer $(O P C)$ represent an understudied cohort of the HPV epidemic. We aimed to investigate the clinical presentation, treatment tolerability and outcomes in patient's $\geq 65$ years old with HPV+ OPC. Patients and Methods: We identified all patients aged 65 and older treated at our Institution with $\mathrm{HPV}+\mathrm{OPC}$ and analyzed patient demographics, disease characteristics, treatment modalities, toxicities, treatment failures, and survival. Charlson comorbidity index was calculated for each patient. Results: 43 patients were identified with a mean age of median age was 70.0 (range 6586). The mean Charlson comorbidity index score for the cohort was 5.2. In total, $72.1 \%$ of patients received what was considered standard-of-care based on stage and pathological features. Nine point three percent of patients required RT-related treatment breaks with the majority being women (75\%). Threeyear actuarial overall survival was $85.5 \%$ (95\% CI: 71.4\%$100 \%)$ and 3-year disease-free survival was $67.3 \%$ (95\% CI: 49.7-91.0\%). Conclusion: This study presented one of the largest series to date evaluating HPV-related OPC in patients $\geq 65$. Elderly individuals with HPV+ OPC have favorable overall survival with high treatment tolerability independent of Charlson co-morbidity score. Elderly patients should be considered for stage-appropriate care with omission of specific therapies based on absolute contraindications and patient preference, but not assumptions regarding tolerability.
\end{abstract}

An increasing incidence of Human Papilloma Virus (HPV) related oropharyngeal squamous cell carcinoma (OPC) has

This article is freely accessible online.

Correspondence to: Richard L. Bakst, MD, 1184 5th Ave, Box 1236, New York, NY 10029, U.S.A. Tel: +1 2122413545, e-mail: richard.bakst@mountsinai.org

Key Words: HPV, elderly, head \& neck cancer. been reported over the past several decades. Epidemiologic studies have shown that HPV+ disease most commonly affects middle-aged, white men $(1,2)$. As a result, studies thus far focus on a younger, healthier population with fewer comorbidities and barriers to treatment with elderly patients being underrepresented in major clinical trials $(3,4)$. However, the incidence, treatment tolerability and outcomes in HPV-related OPC in elderly individuals remains poorly defined. Traditionally it has been thought that HPV-related malignances were rare in this cohort but rates may be higher than previously thought $(5,6)$.

The elderly with head and neck cancer, whose comorbidities often pose treatment challenges, represents a unique cohort of patients. The majority of data in the elderly population is derived from smoking and tobacco-associated OPC, which has been associated with worse clinical outcomes (7-9). In the face of increasing evidence that HPVpositive patients may benefit from treatment deintensification, this cohort would potentially be the ideal population to benefit from a reduction in acute toxicity (10). Given the scarce treatment and outcome data for elderly patients with HPV-related OPC, we aimed to analyze our experience with patients $\geq 65$ years of age.

\section{Patients and Methods}

IRB approval was obtained prior to conducting this retrospective chart review. We identified all patients 65 and older treated at our institution with HPV+ OPC. Patients who did not have PCR confirmation of HPV associated malignancy (i.e. p16 positivity, ISH HPV testing, HPV testing not performed, etc.) were excluded from the analysis. We analyzed patient demographics, disease characteristics, treatment modalities, toxicities and treatment failures when present. Treatment failures were defined as either a local recurrence or distant metastasis of the disease as determined by imaging and biopsy confirmation.

Data variables collected and analyzed include age, gender, HPV subtype, primary site, AJCC stage at diagnosis, alcohol use, smoking history, radiation dose, surgical interventions, chemotherapeutic agents used, past medical history, date of last follow-up, radiation toxicities, obstacles to consistent treatment, 
presence of recurrence/metastasis along with subsequent treatments used and survival status, among others. Kaplan-Meier analysis was used to calculate actuarial overall and disease-free survival.

\section{Results}

Patient and disease characteristics. There were 43 patients included in this study, and the median age was 70.0 (range 65-86) years. The majority of the patients, 32 (74.4\%), were male. More than half of the cohort, 27 patients $(62.8 \%)$, had a history of smoking. Twenty-one patients $(48.8 \%)$ had a significant history of smoking, which was defined as a history of 20 pack-years or more. At the time of OPC diagnosis, 4 patients $(9.3 \%)$ were still smoking. The remainder reported to have quit smoking previously. A history of alcoholism or alcohol abuse was identified in 5 individuals $(11.6 \%)$ of the cohort.

The most common HPV genotype was 16 , which was identified in 36 patients $(83.7 \%)$ of the cohort. Other HPV serotypes observed are shown in Table I. At the time of diagnosis, 26 (60.5\%), were designated as AJCC Stage IVA. The most common primary site was the tonsil present in 24 $(55.8 \%)$ of the cohort. Please refer to Table I for further details regarding patient and disease characteristics.

Patient comorbidities. Past medical histories were collected and used to determine a Charlson Comorbidity Index score for each patient. Higher scores indicating greater comorbidity correlated with greater relative risk of death as expected. The minimum score for any given patient in this study was 4 , as determined by his/her age of at least 65 years and the presence of a solid tumor. The maximum score determined for a given patient was 9. The mean index score for the cohort was 5.27. A specific breakdown is available in Table I.

Treatment. Patients in this study received either surgery-based or radiation-based therapies as the primary modality depending on stage, personal preference and multi-disciplinary tumor board recommendation. A summary of the treatments can be found in Table II. Adjuvant radiation therapy was considered standard-of-care for any patient staged T3/T4 or $\mathrm{N} 2$, or with lymphovascular space invasion, close surgical margins, or perineural invasion. Adjuvant chemo-radiotherapy was considered standard of care for patients with extracapsular extension or positive surgical margins. Mean radiation dose delivered to patients was $61.8 \mathrm{~Gy}$ for adjuvant therapy and 65.9 Gy for definitive treatment in mean fractions of 2.0 for both groups.

We summarized treatments below based on American Joint Committee on Cancer (AJCC) staging. Figure 1 summarizes which patients received standard of care by stage. Table II provides a summary of treatment for surgerybased and radiation-based therapies.
Table I. Patient demographics and disease characteristics.

\begin{tabular}{lc}
\hline Variable & Number of patients $(\%)$ \\
\hline Patient characteristics & \\
Gender & \\
Male & $32(74.4)$ \\
Female & $11(33.3)$ \\
Smoking history & \\
$20+$ pack-years & $21(48.8)$ \\
$0<$ Pack-years<20 & $6(14.0)$ \\
None & $16(37.2)$ \\
Still-smoking at diagnosis & $4(9.3)$ \\
Alcohol abuse & $5(11.6)$ \\
Charlson scores & \\
4 & $11(25.6)$ \\
5 & $17(39.5)$ \\
6 & $11(25.6)$ \\
7 & $1(2.3)$ \\
8 & $2(4.7)$ \\
9 & $1(2.3)$ \\
& \\
Disease characteristics & \\
HPV serotype & \\
16 & $36(83.7)$ \\
33 & $2(4.7)$ \\
35 & $4(9.3)$ \\
Unknown & $1(2.3)$ \\
Primary site & $24(55.8)$ \\
Base of tongue & $13(30.2)$ \\
Oropharynx, unspecified & $6(14.0)$ \\
\hline
\end{tabular}

Stage I $(n=4)$. Four patients were stage I at the time of diagnosis. All of them were HPV 16+ and underwent Transoral Robotic Surgery (TORS) and neck dissection. No adverse features warranting adjuvant therapy were present on final pathologic exam.

Stage II $(n=2)$. Two patients were stage II at time of diagnosis. Both underwent TORS with a neck dissection. One patient (age 76) had LVSI as an adverse prognostic feature but did not receive adjuvant therapy due to physician concerns regarding treatment tolerability.

Stage III $(n=10)$. Eight stage III patients received surgery. Five patients had disease with no adverse features, and did not warrant adjuvant therapy. Two patients warranted adjuvant chemotherapy and radiation for ECE and positive margin respectively but both received adjuvant radiation alone with omission of concurrent chemotherapy secondary to physician concerns regarding treatment tolerability (ages 83 and 76). One patient had LVSI and received adjuvant radiation.

Two patients underwent definitive chemotherapy and radiation with one such patient undergoing induction chemotherapy prior to the start of concurrent treatment. 


\section{Patients receiving standard v. non-standard of care by stage}

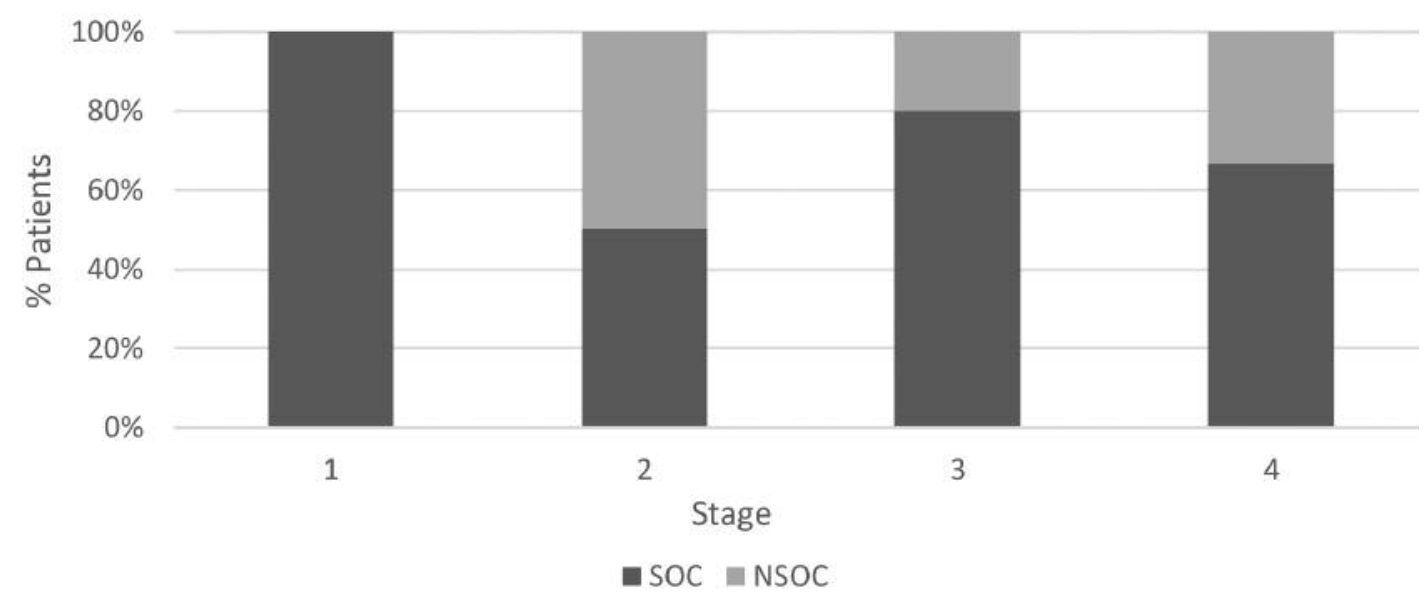

Figure 1. Percentage of patients' standard-of-care by stage. Standard-of-care is defined as whether patients received stage-appropriate care with respect to AJCC stage and adverse pathological features as defined in the Patients and Methods section.

Table II. Description of treatment received by patients within surgery-based and radiation-based groups.

\begin{tabular}{lccc}
\hline Treatment & Number of patients & Average age & Average RT dose (Gy) \\
\hline Surgery & & & -- \\
$\quad$ No adjuvant therapy & 13 & 73.9 & 62.3 \\
$\quad$ Adjuvant RT & 13 & 72.1 & 60.0 \\
$\quad$ Adjuvant ChemoRT & 3 & 65.3 & 66.5 \\
Definitive radiation & & 70.0 & 70.0 \\
$\quad$ Induction chemotherapy & 3 & 66 & 60.0 \\
$\quad$ Concurrent chemotherapy (no induction) & 3 & 80.0 & \\
$\quad$ Radiation alone & 3 & & \\
\hline
\end{tabular}

Stage IVA (n=27). Fifteen patients underwent surgically-based treatment. Six of these patients warranted adjuvant radiation based on adverse features and 6 received it. Nine patients warranted adjuvant chemotherapy and radiation but only 3 received it because of physician concern regarding treatment tolerability $(n=5$; range $70-86)$ and patient refusal $(n=1)$. Of the 9 patients, 4 received just radiation alone and 2 underwent observation because of concern regarding tolerability.

Twelve patients received radiation based therapy. Three patients received radiation alone after being deemed not medically fit for concurrent chemotherapy (age range 79-81 years). Of the 9 patients receiving concurrent chemotherapy (age range 65-78 years), 7 also received induction chemotherapy.

\section{Treatment tolerability}

Surgery. Of the 29 patients receiving surgery, one patient developed a facial subcutaneous emphysema resulting in
PEG placement. There were no post-operative infections or flap-related complications reported. Mean hospital stay was 5.0 days (range 1-27 days).

Chemotherapy. Of the 14 patients receiving concurrent chemotherapy, one developed neutropenic fever, one developed renal toxicity and one developed a related infection. Of the 8 patients receiving induction, one patient developed neutropenic fever. Overall, three patients warranted hospitalization related to neutropenic fever, pulmonary embolism and renal failure.

Radiation. Acutely, $15(34.1 \%)$ patients in this study developed Grade $\geq 3$ mucositis, and 15 patients developed Grade $\geq 2$ xerostomia $(34.1 \%)$. Grade $\geq 3$ dysphagia was found in 12 patients $(27.3 \%)$, and 8 patients $(18.6 \%)$ developed Grade $\geq 3$ dermatitis. PEG tubes were required by 9 patients $(20.5 \%)$ during treatment. All PEG tubes had been 


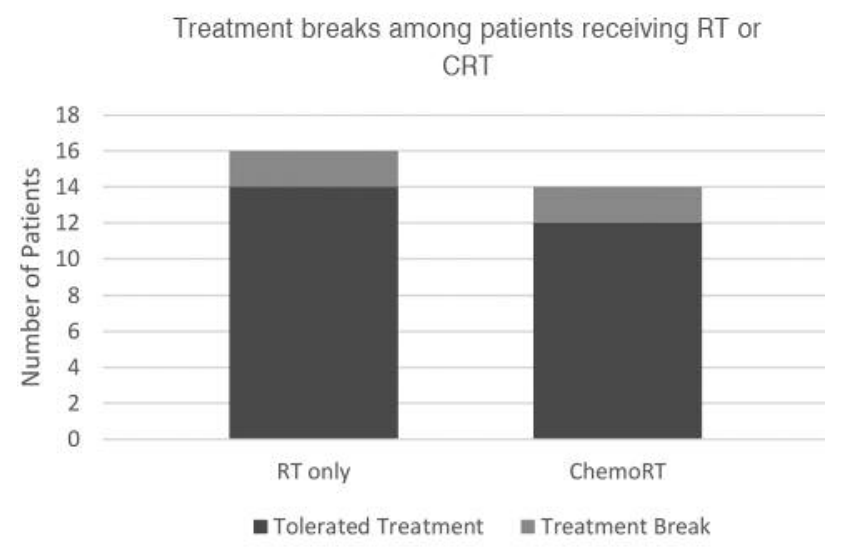

Figure 2. Treatment breaks in patients undergoing definitive or adjuvant radiation $+/$ - concurrent therapy.

removed by 6 months after completion of radiation. Of the 30 patients undergoing radiation, 4 received treatment breaks secondary to treatment toxicity (Figure 2 ). The median age of patients requiring a break was $73(65-81)$ and their average co-morbidity score was 6 .

\section{Treatment and survival outcomes}

Survival. Median follow-up time from the time of diagnosis to the last follow-up appointment for the entire cohort was 24 months. Three-year actuarial overall survival was $85.5 \%$ (95\% CI: 71.4\%-100\%) (Figure 3) and 3-year disease-free survival was $67.3 \%$ (95\% CI: 49.7-91.0\%) (Figure 4). Median survival was 23.1 months (4.7-52.7 months).

Treatment failures. Five (11.3\%) developed a local recurrence and four $(9.1 \%)$ metastases in the lung, bone and abdomen. All recurrences were biopsy proven. All failures developed in either stage III $(\mathrm{N}=3)$ or IV $(\mathrm{N}=6)$ at the time of diagnosis. In the local failure cohort 3 patients had surgery and 2 had definitive radiation. Notably 2 of the 3 patients in this surgical cohort omitted adjuvant radiation and concurrent chemotherapy, which was warranted based on their pathology. In the distant-metastasis cohort, all patients received and completed treatment that was considered standard of care.

\section{Discussion}

Our data represent one of the largest series of elderly patients with HPV-related OPC and demonstrates favorable survival despite advanced age. Furthermore, $72.1 \%$ were able to receive their entire treatment as per standard of care, with only $9.3 \%$ requiring a break from treatment, suggesting that despite significant co-morbidities, this age group tolerated treatment well.

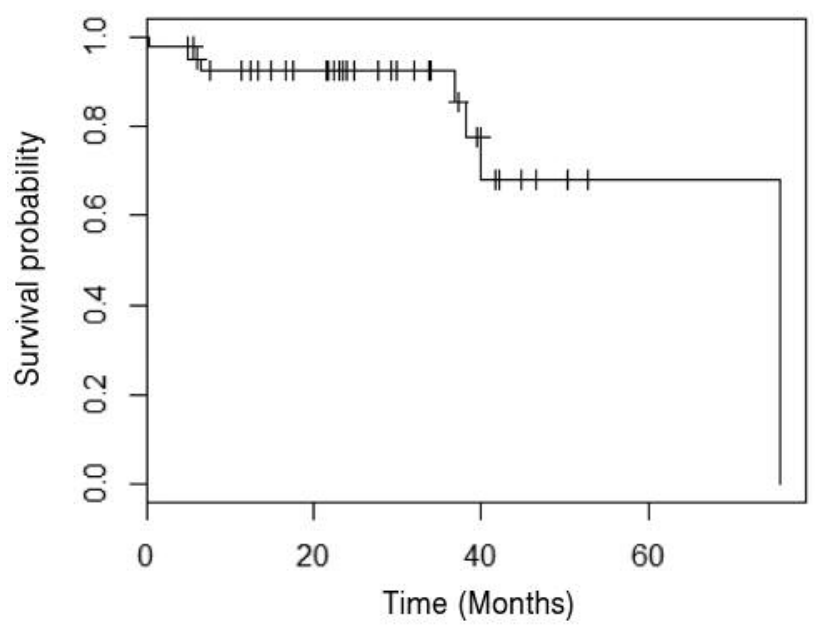

Figure 3. Kaplan-Meier curve for overall actuarial survival for the entire cohort (at risk=43).

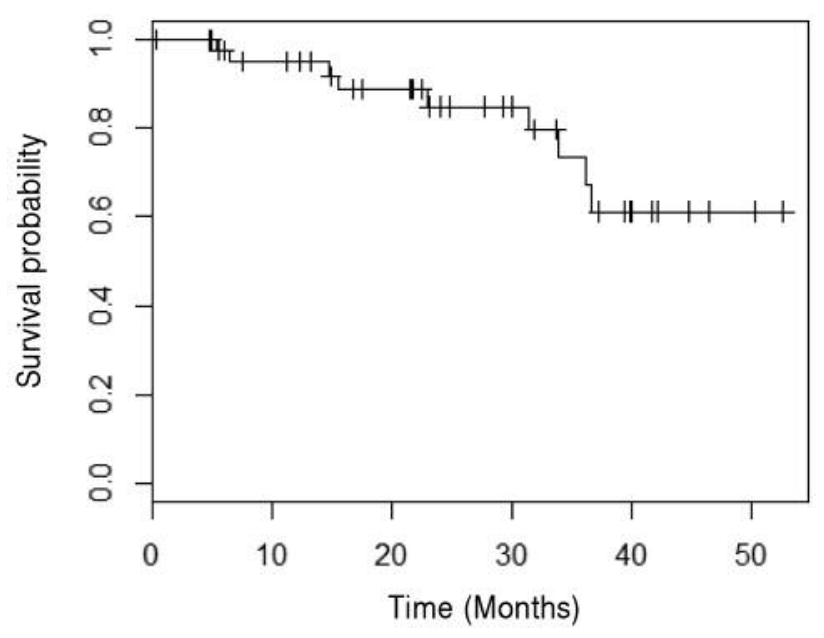

Figure 4. Kaplan-Meier curve for disease-free survival for the entire cohort (at risk=43).

Overall, individuals over age 65 with HPV+ OPC have favorable prognoses and outcomes. Our finding that 3-year actuarial overall survival for elderly HPV+ patients is around $85.5 \%$ is similar to findings from previous studies, which included individuals of all ages (11-13). Notably, our cohort of older individuals had a relatively high incidence of tobacco use, which may negatively alter the diseasecourse. Importantly, $55.6 \%$ of patients who experienced failures had a lifetime history of smoking, and four of these individuals had a pack-year history greater than 20 . Additionally, local failures were mainly seen in patients not undergoing stage or pathologically appropriate care. In the patients who developed a local failure following surgery, the majority did not receive pathologically appropriate 
adjuvant radiation. Therefore, if surgical therapy is planned, it is important to consider the need and potential tolerability of adjuvant radiation. Also, it is helpful to select patients with a low risk of positive margins, as our data indicates that in the elderly population there may be reluctance to undergo adjuvant radiation in the postoperative period.

While the number of subjects in this analysis was limited, females constituted a disproportionate number of patients requiring a treatment break ( $75 \%$ of all radiation breaks) despite a similar average Charlson comorbidity score. It remains unclear as why this was the case; however, it warrants further study. Importantly, none of the patients with the highest Charlson comorbidity scores (greater than or equal to 7) warranted treatment breaks or hospitalizations suggesting that it may not always be a useful surrogate to predict treatment tolerability.

Limitations of this study include its single-institution based, retrospective nature and lack of treatment standardization. Treatment decisions could have been influenced by physician discretion at the time of consult, as well as by patient and family preference. Given the encouraging findings in our experience, further efforts should be made to encourage elderly participation in HPVrelated trials to better understand their disease course. Additionally, quality of life endpoints were not available for this study and would be helpful in weighing the benefits and disadvantages of treatment in this cohort.

While tobacco and ETOH may continue to represent the predominant form of OPC cancer in this cohort, as the HPV epidemic continues the number of elderly related cases will increase. The favorable survival outcomes in this study suggest that the majority of patients with HPV+ OPC will live beyond 3 years. Therefore, whens making treatment decisions, it is important to consider the patient's life expectancy. As the average life expectancy in this country increases, elderly patients should be considered for stageappropriate care with omission of specific therapies based on absolute contraindications and patient preference but not assumptions regarding tolerability or physician bias.

\section{Conclusion}

This represents one of the largest series to date evaluating HPV-related OPC in patients $\geq 65$ years of age. Elderly individuals with HPV+ OPC have favorable survival and treatment outcomes. Treatment tolerability was high and independent of Charlson co-morbidity score.

\section{Conflicts of Interest}

No conflicts of interest to report. This research did not receive any funding from any sources.

\section{References}

1 Pytynia KB, Dahlstrom KR and Sturgis EM: Epidemiology of HPV-associated oropharyngeal cancer. Oral Oncol 50(5): 380386, 2014.

2 Chaturvedi AK, Engels EA, Pfeiffer RM, Hernandez BY, Xiao W, Kim E, Jiang B, Goodman MT, Sibug-Saber M, Cozen W, Liu L, Lynch CF, Wentzensen N, Jordan RC, Altekruse S, Anderson WF, Rosenberg PS and Gillison ML: Human papillomavirus and rising oropharyngeal cancer incidence in the United States. J Clin Oncol 29(32): 4294-4301, 2011.

3 Urban D, Corry J and Rischin D: What is the best treatment for patients with human papillomavirus-positive and -negative oropharyngeal cancer? Cancer 120: 1462-1470, 2014.

4 Maxwell JH, Grandis JR and Ferris RL: HPV-associated head and neck cancer: unique features of epidemiology and clinical management. Annu Rev Med 67: 91-101, 2016.

5 Zumsteg ZS, Cook-Wiens G, Yoshida E, Shiao SL, Lee NY, Mita A, Jeon C, Goodman MT and Ho As: Incidence of oropharyngeal cancer among elderly patients in the united states. JAMA Oncol 2(12): 1617-1623, 2016.

6 Marur S, D'Souza G, Westra WH and Forastiere AA: HPVassociated head and neck cancer: a virus-related cancer epidemic. Lancet Oncol 11: 781-789, 2010.

7 Mehra R, Ang KK and Burtness B: Management of human papillomavirus-positive and human papillomavirus-negative head and neck cancer. Semin Radiat Oncol 22(3): 194-197, 2012.

8 Kumar B, Cordell KG, Lee JS, Worden FP, Prince ME, Tran HH, Wolf GT, Urba SG, Chepeha DB, Teknos TN, Eisbruch A, Tsien CI, Taylor JM, D'Silva NJ, Yang K, Kurnit DM, Bauer JA, Bradford CR and Carey TE: EGFR, p16, HPV Titer, Bcl-xL and p53, sex, and smoking as indicators of response to therapy and survival in oropharyngeal cancer. J Clin Oncol 26(19): 3128-3137, 2008.

9 Kumar B, Cordell KG, Lee JS, Prince ME, Tran HH, Wolf GT, Urba SG, Worden FP, Chepeha DB, Teknos TN, Eisbruch A, Tsien CI, Taylor JM, D'Silva NJ, Yang K, Kurnit DM, Bradford CR and Carey TE: Response to therapy and outcomes in oropharyngeal cancer are associated with biomarkers including human papillomavirus, Epidermal Growth Factor Receptor, Gender, and Smoking. Int J Radiat Oncol 69(2): S109-S111, 2007.

10 Diavolitsis V and Quon H: Treatment de-intensification in HPVassociated oropharyngeal cancer: evidence, controversies, and strategies. Curr Otorhinolaryngol Rep 3(47): 47-55, 2015.

11 Golusinski W, Leemans RC and René DA: HPV Infection in head and neck cancer. Springer International Publishing, 2017.

12 Kimple RJ and Sher DJ: Human papillomavirus and head and neck cancer. Int J Radiat Oncol 92(2): 196-199, 2015.

13 Benson E, Li R, Eisele D and Fakhry C: The clinical impact of HPV tumor status upon head and neck squamous cell carcinomas. Oral Oncol 50: 565-574, 2014 